\title{
A semi coupled shallow water model for vertical velocity distribution in an open channel with submerged flexible vegetation
}

Anupal Baruah ( $\boldsymbol{\nabla}$ anupalbaruah@iitg.ac.in )

Indian Institute of Technology Guwahati

\section{Arup Kumar Sarma}

Indian Institute of Technology Guwahati

Gilbert Hinge

Assam Down Town University

\section{Research Article}

Keywords: Flexible vegetation, Shanon'sEntropy, Shallow water equation, Cantilever beam theory.

Posted Date: May 17th, 2021

DOl: https://doi.org/10.21203/rs.3.rs-344883/v1

License: (c) (1) This work is licensed under a Creative Commons Attribution 4.0 International License.

Read Full License 


\title{
A semi coupled shallow water model for vertical velocity distribution in an open channel with submerged flexible vegetation
}

\author{
Anupal Baruah ${ }^{1}$; Arup Kumar Sarma ${ }^{2}$; Gilbert Hinge ${ }^{3}$ \\ ${ }^{1}$ Research Scholar, Dept. of Civil Engineering, Indian Institute of Technology Guwahati, Guwahati, Assam \\ 781039, India. Email: anupalbaruah@iitg.ac.in \\ ${ }^{2}$ Professor, Dept. of Civil Engineering, Indian Institute of Technology Guwahati, Guwahati, Assam 781039, \\ India (corresponding author). Email: aks@iitg.ac.in \\ ${ }^{3}$ Asst. Professor, Dept. of Civil Engineering, Assam Downtown University. Email: gilberthinge@gmail.com
}

\begin{abstract}
Flow-vegetation interactions modify the instream roughness and flow characteristics in the river and estuaries. This study proposes a new quasi three-dimensional hydrodynamic framework to compute the vertical velocity profile in an open channel having submerged flexible vegetation. A modified form of two-dimensional depth-averaged shallow water equations coupled with vegetal drag forces is derived and applied in the simulation. The explicit second-order accurate TVD McCormack predictor-corrector finite difference method with operator splitting technique is used to solve the governing equations in MATLAB. The TVD approach is robust and gives accurate results free from numerical oscillations. The bending profile of the flexible stems under various flow events is calculated from the cantilever beam theory. The vertical velocity profile in the vegetation layer and the free water layer is estimated from Reynold's stress equation and Shannon's entropy theory. The present model is used to replicate some popular experimental test cases. Results indicate a conservative and robust model performance under different flow conditions and patch density. Quantitative analysis of the predicted results is carried out using two statistical indices and found satisfactory.
\end{abstract}

Keywords: Flexible vegetation, Shanon'sEntropy, Shallow water equation, Cantilever beam theory. 


\section{Introduction}

The floodplains and the riparian vegetations modify the land use-land cover pattern, channel roughness, turbulence intensity, shear stress characteristics, and water quality of a river system (McBride et al. 2007). These alterations, in turn, affect the flow velocity, solute, and sediment transport processes (Salama and Bakry 1992; Wang et al. 2018). The presence of vegetation also impacts the habitat richness and biodiversity in the river ecosystem (Verschoren et al. 2016). Thus, a precise understanding of flow dynamics under the vegetated environment is essential for implementing habitat restoration and conservation strategies in any natural domain (Jalonen and Jarvela 2014; Verschoren et al. 2016).

Based on stem flexibility, vegetations are classified as flexible and rigid vegetation (Aberle and Järvelä 2013). Flexible canopies are ubiquitous and occupy a more extensive section of the flow area in a vegetated terrain than the rigid stems (Armanini et al. 2005). In recent years, flexible vegetation has gained a lot of research interest because of its potential impact on the flow and velocity structure (Huai et al. 2019; van Veelen et al. 2020). Unlike the rigid vegetation, the stem of the flexible vegetations bends to various degrees depending upon the velocity of flow, height, and flexural rigidity(Wilson 2007; She et al. 2014). The different degrees of bending also have a distinctive impact on the flow resistance(Luhar and Nepf 2011). For instance, with the increase in velocity, the flexible vegetation's flow resistance reduces, leading to further reconfiguration of the plants and the vegetation drag(Wu 2008; Shields Jr et al. 2017). Thus, the flow characteristics with flexible vegetations are more complex to deal with as compared to the case of rigid vegetation.

The presence of flexible vegetation, especially under submerged conditions, modifies the flow domain's velocity profile (Nepf 2012). For the non-vegetation channels, the logarithmic expressions are well accepted in defining the vertical velocity profile (Tang 2019). However, with submerged vegetation, the velocity profile becomes more 
complex(Nepf 2012). In those conditions,two-layer or three-layer methods are adopted for velocity distribution above and below the vegetation layer (Keramaris et al. 2015). These methods divided the total water depth into a vegetated region and a free water region and distributed the velocity profiles using different approximations methods (Afzalimehr et al. 2019). For instance, the study carried out by $\mathrm{Pu}$ et al. (2019) divided the flow region into multiple layers and predicts the velocity profile in those layers from mixing length models and eddy viscosity models. Klopstra et al. (1997) proposed a two-layer analytical model to distribute the velocity profile in a vegetated open channel. The model calculates the turbulent stresses in the vegetation region from the Boussinesq hypothesis. Huai et al. (2013) divide the flow regime into a free surface layer, an outer layer, and an inner vegetated layer. Yang and Choi (2010) distributed the velocity profile using a two-layer model in an open channel with immersed vegetation. A large number of studies that attempted to predict the vertical velocity distributions using similar approaches can be found in the literature (Huai et al. 2014; Wang et al. 2015). Though these methods are simple to apply, they have their shortcomings. The eddy viscosity and mixing length models mentioned above fail to capture the anomaly in the flow structure. For instance, near the flow separation regions in a river, the mixing length models fail to predict the velocity dip (Chin and Murray 1992), which is often the case, especially in natural flow domains. These models also require seven to eight parameters, viz shear velocity, energy slope, hydraulic radius, etc., for velocity computation. The difficulties associated with measuring these parameters are another shortcoming of the analytical formulations for field application (Chen and Kao 2011).

The probabilistic approach can tackle these difficulties to obtain the vertical velocity distribution in open channels. Following Chiu and Said (1995), considering the time-average velocity as a probabilistic variable, the velocity distribution may be obtained by maximizing the Shanon's entropy. The velocity distribution obtained from this method has an advantage 
as it required few input parameters and able to describe the velocity distribution in all circumstances. The entropy model distributes the vertical velocity profile at a particular location primarily from the depth-averaged velocity information in a channel cross-section (Kubrak et al. 2008). With this method, the location of maximum velocity can also be obtained. A dimensionless entropy parameter $\mathrm{M}$ can be derived from the mean and maximum velocity information at a channel crossection (Baruah and Sarma 2020). This parameter M represents the channel cross-section, slope, bedform, geometric shape and can characterize the velocity distribution in an open channel flow (Kumbhakar and Ghoshal 2017). The methodology section of this paper provides further details about this method.

The measurement of depth-averaged velocity, particularly in a natural domain, is a tedious task. However, two-dimensional shallow water models are excellent numerical tools to predict the streamwise and transverse velocities even under worst flow events (Blanckaert 2001). The ease of getting the depth-averaged velocity from the shallow water models makes the authors proposing an integrated formulation by linking the entropy formulation with the 2D hydrodynamic models. A full quasi three-dimensional numerical framework can be developed for vertical profile estimation in open channels evidently under a vegetated environment by coupling these two approaches. As per the author's knowledge, no such study has been reported by linking the entropy theory with the shallow water model to compute the vegetated channel flow's vertical velocity profile.

In this work, the authors developed a semi-coupled approach to compute the threedimensional flow structure in a vegetated channel with submerged flexible vegetations at different degrees of submergence and vegetation density. The governing equations are transformed into boundary-fitted coordinates to make the model applicable in complex channel geometry and unsteady flow. TVD Mc-Cormack predictor-corrector finite difference scheme with operator splitting technique is employed here to solve the hyperbolic equations. 
The configuration of the flexible stems under different streamflow is evaluated from the large deflection cantilever beam theory. The main advantage of applying the beam theory in the present work is that it can effectively capture different degrees of stem deflection under high and low flow events. The computed hydraulic parameters from the shallow water model are used in Shannon's entropy theory and Reynolds's stress equation to calculate the vertical velocity in the free water layer and within the vegetation region. The computed outputs are compared with the published experimental results.

\section{2) Numerical model}

\subsection{Derivation of the governing equation and numerical scheme}

In this work, full Saint Venant equations (continuity and momentum equation) are solved in boundary-fitted coordinates. A modified form of the shallow water equation is derived and applied to compute the flow domain's hydrodynamic parameters. The deviatoric form of twodimensional depth-averaged shallow water equation presented by Liang and Borthwick (2009)

$$
\begin{aligned}
& \mathrm{U}=\left\{\begin{array}{c}
\eta \\
\mathrm{hu}
\end{array}\right\} \mathrm{F}=\left\{\begin{array}{c}
\mathrm{hu} \mathrm{u}^{2}+.5 * g *\left(\eta^{2}-2 \eta Z_{b}\right) \\
\mathrm{huv}
\end{array}\right\} \mathrm{G}=\left\{\begin{array}{c}
\mathrm{hv} \\
\mathrm{huv} \\
5 * \mathrm{~g} *\left(\eta^{2}-2 \eta Z_{b}\right)+\mathrm{hv}^{2}
\end{array}\right\} \\
& \mathrm{S} \quad\left\{\begin{array}{c}
0 \\
-\mathrm{g \eta}\left(\frac{d Z_{b}}{d x}-\mathrm{S}_{\mathrm{fx}}\right) \\
-\mathrm{g \eta}\left(\frac{d Z_{b}}{d y}-\mathrm{S}_{\mathrm{fy}}\right)
\end{array}\right\}
\end{aligned}
$$

Where hu and hv are the momentum fluxes in $\mathrm{x}$ and $\mathrm{y}$ direction, $\frac{d Z_{b}}{d x}$ and $\frac{d Z_{b}}{d y}$ are the bed slope in $\mathrm{x}$ and $\mathrm{y}$ direction, $\eta$ is the water surface elevation, $S_{\mathrm{fx}}$ and $\mathrm{S}_{\mathrm{fy}}$ are friction slope in $\mathrm{x}$ and $\mathrm{y}$ direction, $Z_{b}$ is the bed level elevation.

Under the steady-state condition, this formulation is further modified, hereby balancing the flux gradient and the source term in the x-direction momentum equation (equation-1) 


$$
\begin{aligned}
& =\frac{d}{d x}\left(.5 * g *\left(\eta^{2}-2 \eta Z_{b}\right)\right)=-\mathrm{g \eta}\left(\frac{d Z_{b}}{d x}\right) \\
& =\frac{g}{2}\left(\frac{d}{d x}\left(\eta^{2}\right)-\frac{d}{d x}\left(2 \eta Z_{b}\right)\right)=-\mathrm{g \eta}\left(\frac{d Z_{b}}{d x}\right) \\
& =2 \eta \frac{d \eta}{d x}-2\left(\eta \frac{d Z_{b}}{d x}+Z_{b} \frac{d \eta}{d x}\right)=-2 \eta\left(\frac{d Z_{b}}{d x}\right) \\
& =\mathrm{\eta} \frac{d \eta}{d x}-Z_{b} \frac{d \eta}{d x}=0 \\
& =\mathrm{\eta} \frac{d \eta}{d x}=(\eta-h) \frac{d \eta}{d x} \\
& =\mathrm{h} \frac{d \eta}{d x}
\end{aligned}
$$

The above simplification modifies the momentum equation and also the source terms. The expressions with the bed level elevations are omitted from the momentum equations. The inclusion of water surface slope in the gravitational force component provides more flexibility in the model to apply in undulating bed and smooth channel bed.

The governing equation is transformed into a boundary-fitted coordinate to handle the complex channel geometry in the next phase. The modified form of equation (1) is transformed from the physical domain $(x, y)$ to the computational plane $(\mathcal{E}, \eta)$ (Anderson and Wendt 1995). These grid conversions automatically capture the curvilinearity in the flow domain and make the model applicable in any channel geometry. The vegetation effect on the flow dynamics is incorporated by adding the drag force in the source term of the momentum equation

$$
\begin{gathered}
\frac{\partial}{\partial t}[J \eta]+\frac{\partial}{\partial \xi}[J h U]+\frac{\partial}{\partial \eta}[J h V]=0 \\
\frac{\partial}{\partial t}[J h u]+\frac{\partial}{\partial \xi}[J\{h u U\}]+\frac{\partial}{\partial \eta}[J\{h u V\}]=-J g h\left(\frac{d \eta}{d x}-s_{f x}\right)-\frac{F_{D x}}{\rho}
\end{gathered}
$$




$$
\frac{\partial}{\partial t}[J h v]+\frac{\partial}{\partial \xi}[J\{h U v\}]+\frac{\partial}{\partial \eta}[J\{h V v\}]=-J g h\left(\frac{d \eta}{d y}-s_{f y}\right)-\frac{F_{D y}}{\rho}
$$

Where $\eta$ is the water surface elevation $(\mathrm{m}), \mathrm{hu}$ and $\mathrm{hv}$ are the unit discharges in $\mathrm{x}$ and $\mathrm{y}$ direction, $\frac{d \eta}{\partial x}$ and $\frac{d \eta}{\partial y}$ water surface gradients, $\mathrm{h}$ is the flow depth $(\mathrm{m})$ measured from the bed up to the free surface, $s_{f x}$ and $s_{f y}$ are the bed friction components in the longitudinal and transverse direction, $\rho$ is the density of water and $F_{D x}$ and $F_{D y}$ are the drag force due to the vegetation. $\mathrm{U}$ and $\mathrm{V}$ transformed velocity in the computational domain in $\xi, \eta$ coordinate system and expressed as

$$
\begin{array}{cc}
\mathrm{U}=\left(\frac{\partial \xi}{\partial x} u+\frac{\partial \xi}{\partial y} v\right) & \mathrm{V}=\left(\frac{\partial \eta}{\partial x} u+\frac{\partial \eta}{\partial y} v\right) \\
\mathrm{J}=\left|\begin{array}{ll}
\mathrm{x}_{\varepsilon} & \mathrm{x}_{\mathrm{\eta}} \\
\mathrm{y}_{\varepsilon} & \mathrm{y}_{\mathrm{\eta}}
\end{array}\right|=\left|\begin{array}{ll}
\mathrm{x}_{\varepsilon} & \mathrm{y}_{\varepsilon} \\
\mathrm{x}_{\mathrm{\eta}} & \mathrm{y}_{\mathrm{\eta}}
\end{array}\right| \quad \mathrm{s}_{\mathrm{fx}=} \frac{\mathrm{n}^{2} * \mathrm{u} * \operatorname{sqrt}\left(\mathrm{u}^{2}+\mathrm{v}^{2}\right)}{\mathrm{h}^{1 / 3}} \quad \mathrm{~s}_{\mathrm{fy}}=\frac{\left.\mathrm{n}^{2} * \mathrm{v} * \operatorname{sqrt}(\mathrm{u})^{2}+\mathrm{v}^{2}\right)}{\mathrm{h}^{1 / 3}}
\end{array}
$$

The drag force from the vegetation in the $\mathrm{x}$ and $\mathrm{y}$ direction is expressed as

$$
\begin{aligned}
& F_{D x}=\frac{1}{2} * C_{D} * \rho * \mathrm{~m} * A_{v} * u_{v} * \sqrt{u_{v}^{2}+v_{v}^{2}} \\
& F_{D y}=\frac{1}{2} * C_{D} * \rho * \mathrm{~m} * A_{v} * v_{v} * \sqrt{u_{v}^{2}+v_{v}^{2}}
\end{aligned}
$$


In the above expression $A_{v}=$ Projected or frontal area of vegetation, $C_{D}=$ Drag coefficient, $\mathrm{m}$ $=$ vegetation density, $u_{v}$ and $v_{v}$ are the apparent velocities in $\mathrm{x}$ and $\mathrm{y}$-direction. The expression for $u_{v}$ and $v_{v}$ proposed by Stone and Shen (2002) as

$$
u_{v}=\mathrm{u}\left(\frac{h_{v}}{h}\right)^{\frac{1}{2}} \quad ; v_{v}=\mathrm{v}\left(\frac{h_{v}}{h}\right)^{\frac{1}{2}}
$$

In the above expression $h_{v}$ is the height of the flexible vegetation height.

In equation $6(\mathrm{c}, \mathrm{d})$, the coefficients $\mathrm{x}_{\varepsilon}, \mathrm{x}_{\eta}, \mathrm{y}_{\varepsilon}, \mathrm{y}_{\eta}$ are obtained during the grid transformation and calculated using the central finite difference scheme. Jacobean matrix $(\mathrm{J})$ ensures the grid transformation in the domain. The unsteady governing flow equations (3-5) are hyperbolic partial differential equations and difficult to solve analytically. These equations are generally targeted to solve by employing numerical methods viz finite difference, finite volume, and finite element. In this work, the second order accurate explicit Mc-Cormack predictor-corrector scheme (McCormack, 2003) is used to solve the unsteady flow equations. Many researchers have used this method to solve unsteady flow under different situations (Bora and Kalita 2020). This method consists of two steps, namely predictor and corrector. Although the technique is simple for implementation, it often encounters dispersion error in the solution. To avoid that, TVD scheme is added after the corrector step(Kalita 2016).

A splitting algorithm is employed in the solution by dividing the two-dimensional equations into two one-dimensional equations and solved at each time step. Using the operator splitting techniques proposed by Strang(1968), equation (3),(4),(5) can be expressed in matrix form as

$$
\frac{\partial \omega}{\partial t}+\frac{\partial €}{\partial \xi}=R
$$




$$
\frac{\partial \omega}{\partial t}+\frac{\partial \lambda}{\partial \eta}=Q
$$

Where,

$$
\begin{array}{ccccc}
J \eta & & J h U & & J h V \\
J h u & €= & J\{h u U\} & \lambda= & J\{h u V\} \\
J h v & & J\{h U v\} & & J\{h V v\}
\end{array}
$$

0

$$
\begin{gathered}
\mathrm{R}=\quad-J g h\left(\frac{d \eta}{d x}-s_{f x}\right)-\frac{F_{D x}}{\rho} \\
-J g h\left(\frac{d \eta}{d y}\right)
\end{gathered}
$$

0

$$
\begin{array}{r}
\mathrm{Q}= \\
-J g h\left(\frac{d \eta}{d x}\right) \\
-J g h\left(\frac{d \eta}{d y}-s_{f y}\right)-\frac{F_{D y}}{\rho}
\end{array}
$$

$(11 \mathrm{a}, \mathrm{b})$

The equations are solved consecutively in four cycles. In the first sweep, the calculation starts with the prediction of primitive variables in $\xi$ direction equations from the known initial value. The predicted values are corrected in the corrector step. In the second sweep, using the updated variables, the equations are solved in the $\eta$ direction following the predictor and corrector step. Again in the third sweep, the updated variables from the second sweep are used to calculate the new flow variables in $\eta$ direction. Finally, in the fourth sweep the updated variables from the previous cycle are used to compute the flow variables in the next time step. It is mandatory to mention that except for the incoming variables entire computational procedure is the same from sweep-1 to sweep-4. For instance, in $\xi$ direction, sweep 1 uses the initial values, and in sweep -4, updated variables from the previous steps are used in the computation. The detailed procedure of the numerical scheme used in the first 
sweep in $\xi$ direction is presented below. A similar process is adopted in the $\eta$ direction for the next sweep by changing the subscript to $j$.

Predictor-

$$
\omega_{i}^{p}=\omega_{i}^{k}-\frac{\Delta t}{\Delta \xi}\left(€_{i}-€_{i-1}\right)^{k}+\Delta t * R_{i}^{k}
$$

Corrector-

$$
\begin{gathered}
\omega_{i}^{c}=\omega_{i, j}{ }^{k}-\frac{\Delta t}{\Delta \xi}\left(€_{i+1}-€_{i, j}\right)^{p}+\Delta t * R_{i}^{p} \\
\omega_{i, j}^{k+1}=\frac{1}{2}\left(\omega_{i, j}^{p}+\omega_{i, j}{ }^{c}\right)+T V D_{i}
\end{gathered}
$$

Where

$$
T V D_{i}=\left[B\left(\mathrm{r}_{\mathrm{i}}^{+}\right)+B\left(\mathrm{r}_{\mathrm{i}+1}^{-}\right)\right] \cdot \Delta \omega_{i+1 / 2}^{k}-\left[B\left(\mathrm{r}_{\mathrm{i}-1}^{+}\right)+B\left(\mathrm{r}_{\mathrm{i}}^{-}\right)\right] \cdot \Delta \omega_{i-1 / 2}^{k}
$$

In the above equation, the superscript $\mathrm{p}$ and $\mathrm{c}$ indicate the predictor and corrector steps. The computed variables at the known time step are expressed with the superscript $\mathrm{k}$. The variables at the unknown time step are evaluated by averaging the predicted and corrected variables and adding the TVD term $\left(T V D_{i}\right)$. The grid spacing and time interval is denoted by $\Delta \xi$ and $\Delta t$.In equation 15 ,

$$
\begin{aligned}
& \Delta \omega_{i+1 / 2}=\omega_{i+1}-\omega_{i} \\
& \Delta \omega_{i-1 / 2}=\omega_{i}-\omega_{i-1}
\end{aligned}
$$

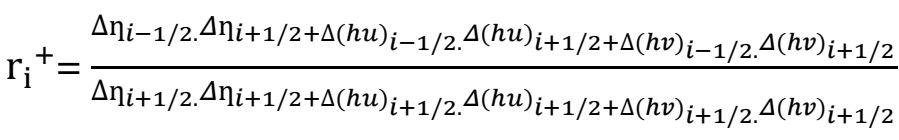




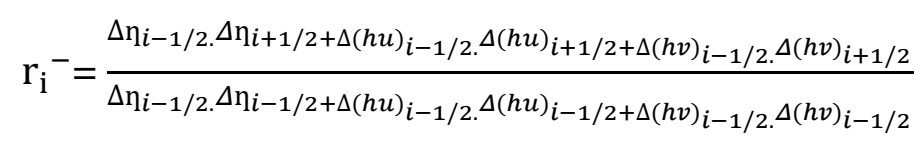

The function B () in equation (15) is defined as

$\mathrm{B}(\mathrm{x})=0.5^{*} \mathrm{C}^{*}[1-\phi(\mathrm{x})]$ and super bee flux limiter function is given as

$\Phi(\mathrm{x})=\max (0, \min (2 \mathrm{x}, 1))$

Where $\mathrm{C}=\mathrm{f}\left(C_{r}\right), C_{r}$ is the courant number given as $C_{r}=\frac{\Delta t *\left(\left(u * \operatorname{sqrt}\left(u^{2}+v^{2}\right)+\sqrt{g * h}\right)\right)}{\Delta x}$

\subsection{Deflection of the flexible vegetation}

The variation in the upstream water levels at different flow events influences the curve length of the flexible stems. Under high flow events the load on the stem increases leading to the higher degree of reconfiguration and vice versa. The projected deflection height of the flexible stem is estimated from the larger deflection cantilever beam approach by assuming that vegetation gravity and buoyant force due to water is negligible(Whittaker et al. 2015). In figure $1, \mathrm{z}$ coordinates represent the distance from the bed to the top of the vegetation; $\mathrm{L}$ is flexible vegetation height before bending and $h_{v}$ is the projected deflection height. The representative vertical velocity profile in an open channel with submerged flexible vegetation is shown in figure-2.

Imposedaverage load $\mathrm{P}$ on flexible stems due to the upstream water head is expressed as (Huai et al. 2013)

$$
\mathrm{P}=\frac{\rho g \mathrm{~s}_{\mathrm{f}} H}{m}
$$

Where $\mathrm{m}$ is the vegetation density per unit area, $\mathrm{s}_{\mathrm{f}}$ is the energy slope, $\rho$ is the density of water $\left(\mathrm{KN} / \mathrm{m}^{3}\right), \mathrm{H}$ is the total flow depth $(\mathrm{m}), \theta$ is the angle of rotation after bending,EI is the 
flexural rigidity $\left(\mathrm{N} . \mathrm{m}^{2}\right)$ and $\mathrm{g}$ is the acceleration due to gravity. The curve length $(\mathrm{S})$ of the element after bending is found from (Huai et al. 2013)

$$
\mathrm{S}=\int_{0}^{h_{v}} \sqrt{\frac{d z}{1-\left[\left(\frac{P}{2 E I}\right) *\left(\frac{z^{3}}{3 h_{v}}-z^{2}+z h_{v}\right)\right]^{2}}}
$$

Where $\mathrm{h}_{\mathrm{v}}=$ height of the vegetation $(\mathrm{m})$ and $\mathrm{z}=$ any depth along the vertical $(\mathrm{m})$

\subsection{Velocity distribution in the vegetated layer}

In the vegetation layer velocity profile is estimated from the momentum equation by applying the force balancing between the Reynolds shear stress, vegetation roughness and vegetation roughness

$$
\frac{d \tau}{d z}+\rho g S_{f}-\frac{d F_{x}}{d z}=0
$$

Net Resultant force $\left(F_{x}\right)$ on the bending vegetation is evaluated from the drag force and the friction force component, and finally the velocity profile in the vegetation layer is expressed as (Huai et al. 2013)

$$
\alpha=\sqrt{\frac{C_{d} m D}{0.3\left(H-h_{v}\right)}}
$$

$\boldsymbol{U}$

$$
=\frac{2 g S_{f}\left\{\alpha * h * \exp \left(\alpha *\left(z-h_{v}\right)\right)+1\right\}}{\sqrt{m D\left\{C_{d}\left[1-\left(\frac{P}{2 E I}\right)^{2}\left(\frac{z^{3}}{3 h_{v}}-z^{2}+z h_{v}\right)^{2}\right]+C_{f} * C_{p}\left(\left(\frac{P}{2 E I}\right)\left(\frac{z^{3}}{3 h_{v}}-z^{2}+z h_{v}\right)^{3}\right) / \sqrt{\left.1-\left[\left(\frac{P}{2 E I}\right)\left(\frac{z^{3}}{3 h_{v}}-z^{2}+z h_{v}\right)^{2}\right]\right\}}\right.}}
$$


At $z=h_{v}$

$$
u_{v}=\frac{2 S_{f}(\alpha h+1)}{\sqrt{m D\left\{C_{d}\left[1-\left(\frac{P_{b}}{6 E I}\right)^{2}\right]+C_{f} C_{p}\left(\left(\frac{P_{v}{ }^{2}}{6 E I}\right)^{3}\right) / \sqrt{1-\left[\left(\frac{\mathrm{Ph}_{v}{ }^{2}}{6 E I}\right)^{2}\right]}\right.}}
$$

$C_{d}=$ Drag coefficient, $C_{p}=$ perimeter of the stem cross-section and $C_{f}=$ friction coefficient, $\alpha=$ Constant relating the hydraulic and vegetation characteristic.

\subsection{Shannon's entropy theory for velocity distribution in the free water layer}

The average and maximum velocity in a channel cross-section can be related to the entropy model as

$$
\phi(M)=\left(\frac{e^{M}}{e^{M}-1}-\frac{1}{M}\right)=\frac{u_{\text {avg }}}{u_{\max }}
$$

Where, $M=$ Dimensionless entropy parameter, $u_{\max }=$ maximum velocity in the velocity profile, $u_{\text {avg }}=$ depth averaged velocity, $y_{\max }=$ Location of maximum velocity in the vertical sample above the vegetation, $y_{0}=$ water depth from bottom to the top of the vegetation, and $\phi(M)$ is a function of entropy parameter.

Chiu C L (1989) applied the principle of maximum entropy to the Shannon entropy (Shannon 1948) to obtain the following expression for vertical velocity distribution.

$$
\frac{u}{u_{\max }}=\frac{1}{M} \ln \left[1+\left(e^{M}-1\right) \frac{\beta-\beta_{o}}{\beta_{\max }-\beta_{o}}\right]
$$

Where $u$ is the velocity at any point in a vertical, $u_{\max }$ is the maximum velocity occurring in that vertical, and $M$ is the entropic parameter. For the convenience of representing the flow, a special coordinate system was defined such that the velocity is dependent on $\beta$. The velocity is assumed to increase from zero $\left(\beta=\beta_{o}\right)$ to a maximum value $u_{\max }\left(\beta_{\max }=\beta_{o}\right)$. Therefore, $u$ is a function of $\beta$. 


\subsection{Initial and Boundary condition}

The initial and boundary conditions are essential for the smooth functioning of the numerical model. In the hydrodynamic simulation, a discharge value is provided as the initial condition. The solid walls are simulated as a no-slip boundary (Anderson and Wendt 1995) viz both the streamwise and transverse velocity components are assigned with a zero value. At the upstream boundary, constant discharge and the flow depth corresponding to that discharge are set at the downstream boundary.

\subsection{Semi coupled approach}

The present study used the Semi-Coupled approach to link the vertical velocity distribution model and the 2-D hydrodynamic model presented in the earlier section. A full quasi threedimensional numerical model was developed by coupling these two, which is used for estimation of vertical profile in open channels having submerged flexible vegetation. Figure 3 shows the flowchart of the author's proposed model.

Before running the model, the flow domain is discretized into finite-difference grid points both in the lateral and longitudinal direction by solving a set of elliptic partial differential equations. With these grid points, the model begins by setting an initial value of the primitive variable to the generated grids. The present model incorporates the vegetation drag force term explicitly within the hydrodynamic model to evaluate the flow dynamics' vegetation effect. With these initial conditions, the momentum fluxes along the longitudinal and transverse directions are computed at the next time step. The vegetation drag force calculation is exempted from the momentum equation in the non-vegetation regions. The hydrodynamic model runs till steady-state. The model stability criteria are checked from the Courant number. According to this criterion, if the Courant number is less or equal to one, then the 
model is considered to be stable. If this criterion is not satisfied, the time step is readjusted, and the preceding iterations are repeated.

In the second phase, the computed flow outputs from the hydrodynamic model are used in the velocity distribution equations to estimate the vegetated flow region's vertical velocity profile. In order to do that, the entire flow region is divided into two flow layers, viz. the vegetation layer and the free water layer. The steady-state flow depth from the hydrodynamic model is substituted in equation-21 for imposed load calculation over the flexible stem. Once the load is calculated, the curve length $(S)$ of the stem is calculated iteratively from equation22. For validation purposes, the model computed bending profile of the flexible stem is compared with the measured data. A small amount of error $(\varphi=.00001)$ is assigned during the calculation, and the iteration continues till it satisfies the criteria. Then the vegetation characteristics such as patch density $(\mathrm{m})$, stem diameter(D), drag coefficient $\left(C_{D}\right)$, bend vegetation height $\left(h_{v}\right)$ and flexural rigidity (EI) are substituted in equation (25) and (26) to compute the velocity profile within the vegetation zone and at the crown of the vegetation. Further, the calculated velocity at the crown is used in the free layer's velocity distribution. Whereas for the top free water layer, the entropy parameter for the channel cross section is calculated from the depth-averaged velocity and the maximum velocity. The depth-averaged velocity is obtained from the shallow water model and used in equation (27) to calculate $\varnothing$ (M) and the entropy parameter (M) in the channel cross-section. The velocity profile (u) at different depths (z) above the vegetation region to the free water surface is obtained from equation-28.

\section{3) Application of the numerical model}

The performance of the present model is assessed by setting up the model with series of experimental test cases carried out by Amreeva and Kurbak (2007). The model computed 
output is then compared with the experimental outputs reported in their laboratory flume experiment. Amreeva and Kurbak (2007) conducted the experiments in the hydraulic laboratory containing vegetation without foliage in the department of Hydraulic structure, Warsaw Agricultural University. The experiment was performed in a glass-walled flume of $16 \mathrm{~m}$ length, $0.58 \mathrm{~m}$ wide, and $0.6 \mathrm{~m}$ depth. In their experiment, the cylindrical stems of elliptical cross-sections having diameter $\mathrm{d} 1=.00095 \mathrm{~m}$ and $\mathrm{d} 2=.0007 \mathrm{~m}$ were placed in a removal plate of $3 \mathrm{~m}$ length, $.58 \mathrm{~m}$ wide made of PVC. The longitudinal and transverse velocity profiles were measured using a programmable electromagnetic liquid velocity meter. The schematic diagram of the experimental channel is available in (Amreeva and Kurbak 2007). The details of the different test runs of the experimental flume are enlisted in Table- 1 .

In the present study, for numerical simulation, the entire domain is discretized into 191 finite-difference grid points. The grid spacing in the channel is taken as $\Delta x=0.5 \mathrm{~m}$ and $\Delta y=0.1 \mathrm{~m}$. However, in the vegetated region, the stems are placed at a more refined grid spacing. The Mannings or Chezy's roughness coefficient is an essential parameter for shallow water simulations. These coefficients are generally estimated from the mean diameter of the bed material. Amreeva and Kurbak (2007) expressed the bed roughness in terms of equivalent sand grain roughness in his experimental work. Thus, following Marriott and Jayratne (2010), the given equivalent sand grain roughness $\left(k_{s}=.0001, .0018, .0150\right)$ are converted to manning's roughness parameter (n), and it ranges between .008-.018. The discharge in the channel varies from $.0333-.0751 \mathrm{~m}^{3} / \mathrm{sec}$. These flowrates are provided as the upstream boundary condition. The time step is considered as 0.35 second, which gives a courant number of 0.93 . The volume fraction of vegetation (Neph) is incorporated in the drag force calculation, which ranges between $0.32-1.558$. The grids point having no vegetation are exempted from the drag force computation. 
The hydrodynamic simulation provides the flow and velocity information at different discharge and at different locations in the channel .The computed velocity profiles from the shallow water model for the experiment number 1.2.1 and 4.1.1 is shown in Figure 4. The velocity profile from the above figures indicates that the maximum velocity lies between .09$.14 \mathrm{~m} / \mathrm{sec}$ for the discharges $0.0422 \mathrm{~m}^{3} / \mathrm{sec}$ and $0.0693 \mathrm{~m}^{3} / \mathrm{sec}$. The velocity within the vegetation area is found as $0.01 \mathrm{~m} / \mathrm{sec}$ to $0.03 \mathrm{~m} / \mathrm{sec}$. The drag force influence on the flow profiles is clearly observed in the vegetation region. The velocity within the vegetation region progressively reduces at a higher density $(\mathrm{m}=10000)$ compared to the lower vegetation density $(m=2500)$. The imposed load on the flexible stem is computed for different flow events. The bending profile of the flexible stem under the influence of different loads is calculated and compared with the measured results. Figure-5 shows the correlation coefficient between the computed and measured bending profiles of the flexible stems and found satisfactory. The vertical velocity within the vegetation layer and at the crown of the stem is calculated using the procedure mentioned in section 2.5.However, in the free water layer, the velocity profile is estimated from the entropy theory. As mentioned before, the integration of entropy theory with the shallow water model provides a simplified approach for vertical velocity distribution in open channels. The channel entropy parameter is calculated from the computed depth-averaged velocities obtained from the numerical model and the maximum velocity information from the experimental study. The experimental outputs show that maximum velocity appears at the free surface in all the cases

.For illustrating the velocity distribution model, we are considering experiment no 4.1.1. The depth-averaged velocities from the hydrodynamic simulation are found between .01-.015 $\mathrm{m} / \mathrm{sec}$, and the maximum velocity is $0.92 \mathrm{~m} / \mathrm{sec}$. From these two values, $\varnothing(\mathrm{M})$ and the entropy parameter (M) are calculated. The velocity at the crown of the stem is found as $0.67 \mathrm{~m} / \mathrm{sec}$. The free water depth is calculated by subtracting the vegetation height from the 
total water column depth. From the known $\mathrm{M}$ value, the velocity at the crown of the flexible stem and the maximum velocity, the vertical profile is distributed in the free water layer.

The computed vertical velocity profiles at the vegetated portions for different experiments are presented in figure-6. The figure shows that the present model excellently predicts the velocity profiles under different flow events. The discrepancy in the observed and computed results is checked using two statistical performance measures Viz. Root mean square error (RMSE) and mean square error (MSE).

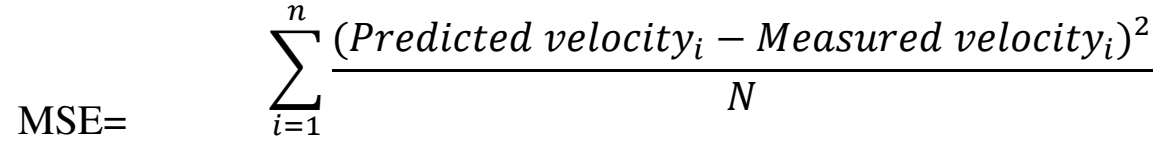

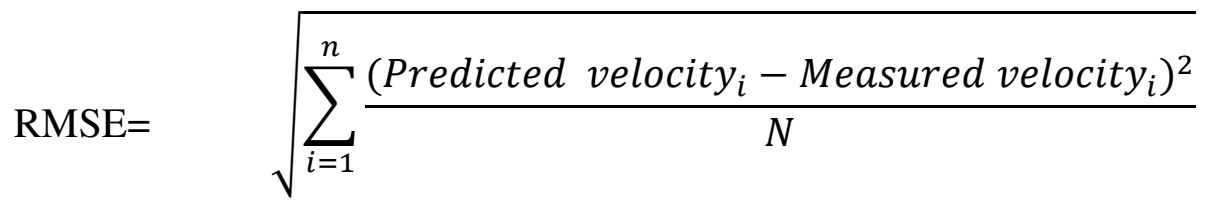

The results obtained based on these measures are shown in Table 2. The values indicate that the proposed model can be used for estimation of the vertical velocity profile in an open channel having submerged flexible vegetations.

These results also indirectly signify the validation of the hydrodynamic model. The entropy model performance depends on the accuracy of the hydrodynamic model's flow parameter. As explained in the earlier section, the depth-average velocity obtained from the shallow water models is fed as an input into the entropy model to predict the velocity profile. With this input, it is observed that the entropy model accurately predicts the velocity profile, which could only be possible if the output flow parameters from the hydrodynamic model are accurate. With this argument, we can say with conviction that the hydrodynamic model is also successfully validated. 


\section{4) Conclusion}

The integration of entropy theory with the shallow water model provides a simplified yet effective approach for vertical velocity distribution in open channels as it requires only the depth averaged velocity value which is precisely predicted from the shallow water equations.This study presents a quasi-three-dimensionalunsteady model framework for vertical velocity distribution in vegetated environment. The model is developed by coupling the vegetation drag force with the modified form of two-dimensional shallow water equations. The governing equations are solved by efficient TVD Mac-Cormack predictor corrector scheme with operator splitting technique in boundary fitted coordinate. The computed depth averaged velocities from the shallow water model is used in the vertical profiles computation at different flow events. In this study, the entropy theory and the Reynolds's stress equation is employed in the computation of vertical flow structure. The applicability of the present semi coupled model is checked in a rectangular channel with submerged flexible vegetations. The vertical velocity profiles from the experimental results are reported by Kurbak et al. (2008). The results indicate that the present model precisely estimate the vertical velocity profile in the vegetated region. This present approach can effectively integrate into river restoration projects, habitat modelling, and river ecology studies to provide useful insight into the determination of velocity profile under vegetated channel flow. However, the flow-vegetation-sediment nexus is beyond the scope of the present work. The model can be further improved by incorporating the morphological influences in the hydrodynamic simulations under the vegetated environment.

\section{Declaration}

Funding: No external funding is received in this study 
Conflict of interest: None

Code availability: The code used in this study is developed in MATLAB

\section{Data availability statement}

The data that support the finding of this study can be found in

https://doi.org/10.1623/hysj.53.4.905

Author contribution:All authors contributed to the study conception and design. Material preparation, data collection and analysis were performed by AnupalBaruah. The first draft of the manuscript was written by Anupal Baruah and all authors commented on previous versions of the manuscript. All authors read and approved the final manuscript. 


\section{Reference}

Aberle J, Järvelä J (2013) Flow resistance of emergent rigid and flexible floodplain vegetation. J Hydraul Res 51:33-45

Afzalimehr H, Maddahi MR, Sui J, Rahimpour M (2019) Impacts of vegetation over bedforms on flow characteristics in gravel-bed rivers. J Hydrodyn 31:986-998

Amreeva G, Kurbak A (2007) Experimental studies on the dimensional properties of half Milano and Milano rib fabrics. Text Res J 77:151-160

Anderson JD, Wendt J (1995) Computational fluid dynamics. Springer

Armanini A, Righetti M, Grisenti P (2005) Direct measurement of vegetation resistance in prototype scale. J Hydraul Res 43:481-487

Baruah A, Sarma AK (2020) A quasi three dimensional hydrodynamic model for velocity distribution in open channel. ISH J Hydraul Eng 1-9

Blanckaert K (2001) Bend-flow simulation using 2D depth-averaged model. J Hydraul Eng $127: 167-170$

Bora K, Kalita HM (2020) A Semi-Coupled Model for Morphological Simulation in River Bend. J Appl Fluid Mech 13:

Chen Y, Kao S (2011) Velocity distribution in open channels with submerged aquatic plant. Hydrol Process 25:2009-2017

Chin C-L, Murray DW (1992) Variation of velocity distribution along nonuniform openchannel flow. J Hydraul Eng 118:989-1001

Chiu C-L (1989) Velocity distribution in open channel flow. J Hydraul Eng 115:576-594

Chiu C-L, Said CAA (1995) Maximum and mean velocities and entropy in open-channel flow. J Hydraul Eng 121:26-35

Huai W, Wang W, Hu Y, et al (2014) Analytical model of the mean velocity distribution in an open channel with double-layered rigid vegetation. Adv Water Resour 69:106-113 
Huai W, Wang W, Zeng Y (2013) Two-layer model for open channel flow with submerged flexible vegetation. J Hydraul Res 51:708-718

Huai W, Zhang J, Katul GG, et al (2019) The structure of turbulent flow through submerged flexible vegetation. J Hydrodyn 31:274-292

Jalonen J, Jarvela J (2014) Estimation of drag forces caused by natural woody vegetation of different scales. J Hydrodyn Ser B 26:608-623

Kalita HM (2016) A new total variation diminishing predictor corrector approach for twodimensional shallow water flow. Water Resour Manag 30:1481-1497

Keramaris E, Pechlivanidis GI, Pechlivanidis IG (2015) The different impact of a halfseparated gravel and vegetated bed in open channels. Environ Process 2:123-132

Klopstra, D., Barneveld, H. J., Van Noortwijk, J. M., \& Van Velzen, E. H. (1996) Analytical model for hydraulic roughness of submerged vegetation. In Proceedings of the congressinternational association for hydraulic research (pp. 775-780). LOCAL ORGANIZING COMMITTEE OF THE XXV CONGRESS.

Kubrak E, Kubrak J, ROWIŃSKI PM (2008) Vertical velocity distributions through and above submerged, flexible vegetation. Hydrol Sci J 53:905-920

Kumbhakar M, Ghoshal K (2017) One-dimensional velocity distribution in open channels using Renyi entropy. Stoch Environ Res Risk Assess 31:949-959

Liang Q, Borthwick AGL (2009) Adaptive quadtree simulation of shallow flows with wetdry fronts over complex topography. Comput Fluids 38:221-234

Luhar M, Nepf HM (2011) Flow-induced reconfiguration of buoyant and flexible aquatic vegetation. Limnol Oceanogr 56:2003-2017

Marriott, M., \& Jayaratne, R. (2010) Hydraulic roughness-links between Manning's coefficient, Nikuradse's equivalent sand roughness and bed grain size. Advances in Computing and Technology 2010, 27-32. 
McBride M, Hession WC, Rizzo DM, Thompson DM (2007) The influence of riparian vegetation on near-bank turbulence: A flume experiment. Earth Surf Process Landforms J Br Geomorphol Res Gr 32:2019-2037

Nepf HM (2012) Flow and transport in regions with aquatic vegetation. Annu Rev Fluid Mech 44:123-142

Pu JH, Hussain A, Guo Y, et al (2019) Submerged flexible vegetation impact on open channel flow velocity distribution: An analytical modelling study on drag and friction. Water Sci Eng 12:121-128

Salama MM, Bakry MF (1992) Design of earthen vegetated open channels. Water Resour Manag 6:149-159

Shannon CE (1948) A mathematical theory of communication. Bell Syst Tech J 27:379-423

She D, Liu D, Xia Y, Shao M (2014) Modeling effects of land use and vegetation density on soil water dynamics: implications on water resource management. Water Resour Manag 28:2063-2076

Shields Jr FD, Coulton KG, Nepf H (2017) Representation of vegetation in two-dimensional hydrodynamic models. J Hydraul Eng 143:2517002

Strang, G. (1968). On the construction and comparison of difference schemes. SIAM journal on numerical analysis, 5(3), 506-517.

Tang X (2019) An improved analytical model for vertical velocity distribution of vegetated channel flows. J Geosci Environ Prot 7:42-60

van Veelen TJ, Fairchild TP, Reeve DE, Karunarathna H (2020) Experimental study on vegetation flexibility as control parameter for wave damping and velocity structure. Coast Eng 157:103648

Verschoren V, Meire D, Schoelynck J, et al (2016) Resistance and reconfiguration of natural flexible submerged vegetation in hydrodynamic river modelling. Environ Fluid Mech 
$16: 245-265$

Wang W, Huai W, Zeng Y, Zhou J (2015) Analytical solution of velocity distribution for flow through submerged large deflection flexible vegetation. Appl Math Mech 36:107120

Wang W, Peng W, Huai W, et al (2018) Roughness height of submerged vegetation in flow based on spatial structure. J Hydrodyn 30:754-757

Whittaker P, Wilson CAME, Aberle J (2015) An improved Cauchy number approach for predicting the drag and reconfiguration of flexible vegetation. Adv Water Resour 83:2835

Wilson C (2007) Flow resistance models for flexible submerged vegetation. J Hydrol $342: 213-222$

Wu F (2008) Characteristics of flow resistance in open channels with non-submerged rigid vegetation. J Hydrodyn Ser B 20:239-245

Yang W, Choi S-U (2010) A two-layer approach for depth-limited open-channel flows with submerged vegetation. J Hydraul Res 48:466-475 


\section{LIST OF TABLES}

Table 1: Flow and vegetation parameters for simulation

\begin{tabular}{|c|c|c|c|c|c|c|c|}
\hline $\begin{array}{c}\text { Experiment } \\
\text { number }\end{array}$ & 1.2 .1 & 1.1 .3 & 2.1 .1 & 2.2 .1 & 3.1 .1 & 3.2 .1 & 4.2 .1 \\
\hline $\begin{array}{c}\text { Flow rate, } \\
\mathbf{Q}\left(m^{3} / \text { sec }\right)\end{array}$ & .0422 & .0333 & .0525 & .0751 & .0605 & .0693 & .0693 \\
\hline $\begin{array}{c}\text { Water depth, } \\
\text { H(m) }\end{array}$ & .2236 & .2475 & .2386 & .2131 & .2386 & .1962 & 2077 \\
\hline $\begin{array}{c}\text { Vegetation } \\
\text { height, } h_{v}(\mathbf{m})\end{array}$ & .161 & .164 & .153 & .132 & .151 & .132 & .138 \\
\hline $\begin{array}{l}\text { Bottom slope } \\
\text { (S) }(\%)\end{array}$ & 8.7 & 8.7 & 8.7 & 17.4 & 8.7 & 17.4 & 17.4 \\
\hline $\begin{array}{l}\text { Drag coefficient } \\
\qquad\left(C_{d}\right)\end{array}$ & 1.4 & 1.4 & 1.4 & 1.4 & 1.4 & 1.4 & 1.4 \\
\hline $\begin{array}{l}\text { Equivalent sand } \\
\text { grain roughness } \\
\qquad\left(k_{s}\right)(\mathrm{m})\end{array}$ & .0001 & .0001 & .0001 & .0001 & .0018 & .0018 & .0150 \\
\hline $\begin{array}{l}\text { Friction } \\
\text { coefficient } \\
\left(C_{f}\right)\end{array}$ & .4 & .4 & .4 & .4 & .4 & .4 & .4 \\
\hline $\begin{array}{c}\text { Vegetation } \\
\text { density } \\
\left(m^{-2}\right)\end{array}$ & 10000 & 10000 & 2500 & 2500 & 2500 & 2500 & 2500 \\
\hline $\begin{array}{c}\text { Flexural rigidity } \\
\text { (EI) } \\
\left(\mathrm{Nm}^{-2}\right)\end{array}$ & $\begin{array}{c}5.81 \\
\times 10^{-5}\end{array}$ & $\begin{array}{c}5.81 \\
\times 10^{-5}\end{array}$ & $\begin{array}{c}5.81 \\
\mathrm{x} 10^{-5}\end{array}$ & $\begin{array}{c}5.81 \\
\times 10^{-5}\end{array}$ & $\begin{array}{c}5.81 \\
\mathrm{x} 10^{-5}\end{array}$ & $\begin{array}{c}5.81 \\
\mathrm{x} 10^{-5}\end{array}$ & $\begin{array}{c}5.81 \\
\times 10^{-5}\end{array}$ \\
\hline
\end{tabular}


Table-2: RMSE and MSE of the computed and observed results

\begin{tabular}{ccc}
\hline Experiment number & \multicolumn{2}{c}{ Statistical Index } \\
\cline { 2 - 3 } & RMSE & MSE \\
\hline 1.2 .1 & 0.0367 & 0.0013 \\
1.1 .3 & 0.0335 & 0.0011 \\
2.1 .1 & 0.0567 & 0.0031 \\
2.2 .1 & 0.0479 & 0.0022 \\
3.1 .1 & 0.0052 & 0.0026 \\
3.2 .1 & 0.0159 & 0.0002 \\
4.1 .1 & 0.028 & 0.0008 \\
\hline
\end{tabular}




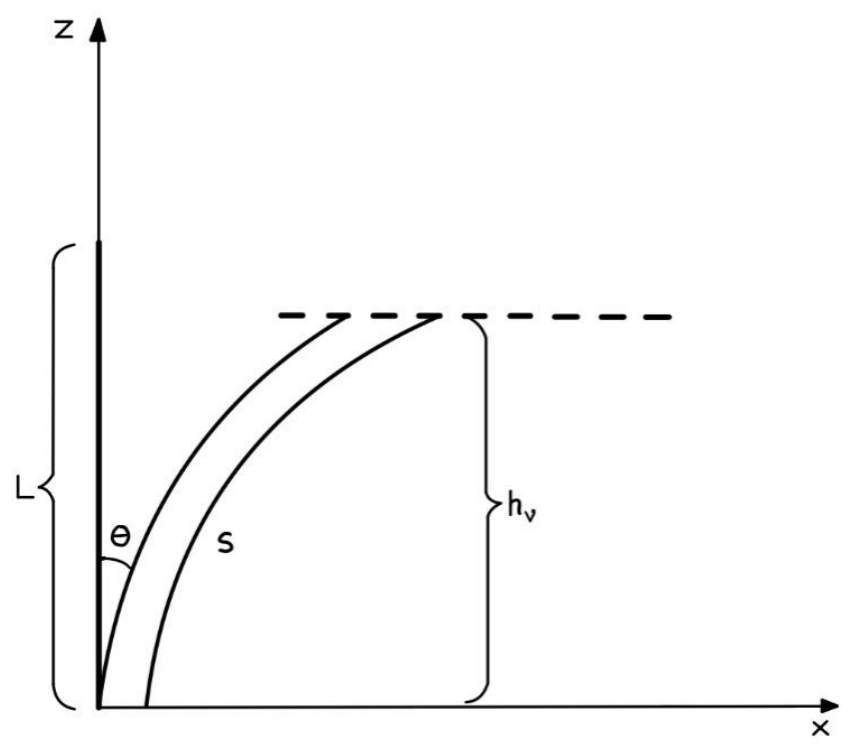

Figure 1: Deflection of Flexible Vegetation (Huai et al. 2013)

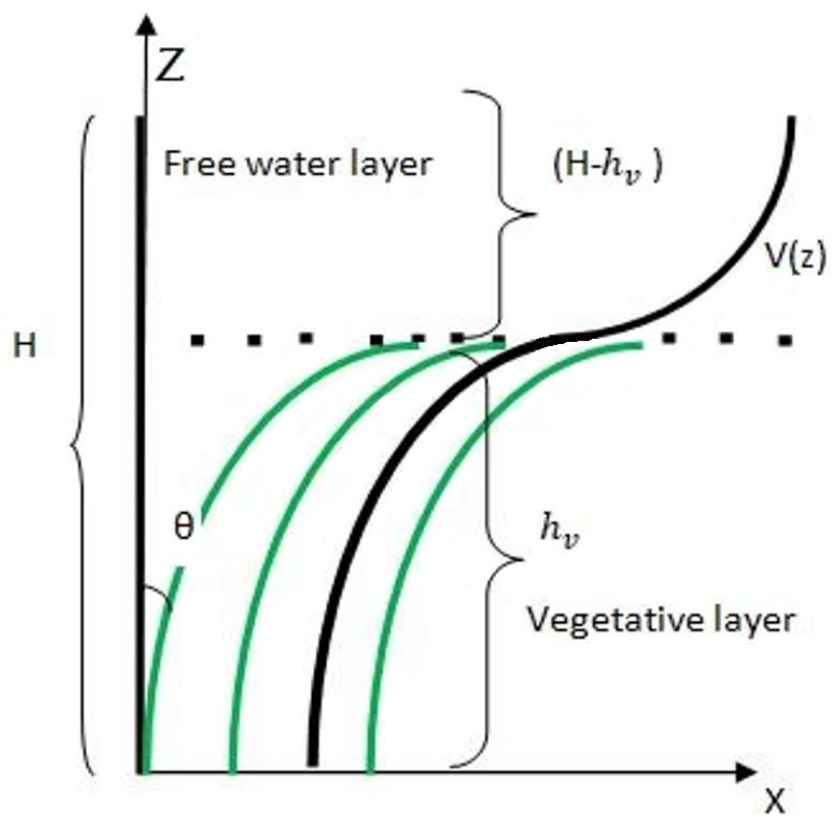

Figure 2: Velocity profile in an open channel with submerged flexible vegetation 


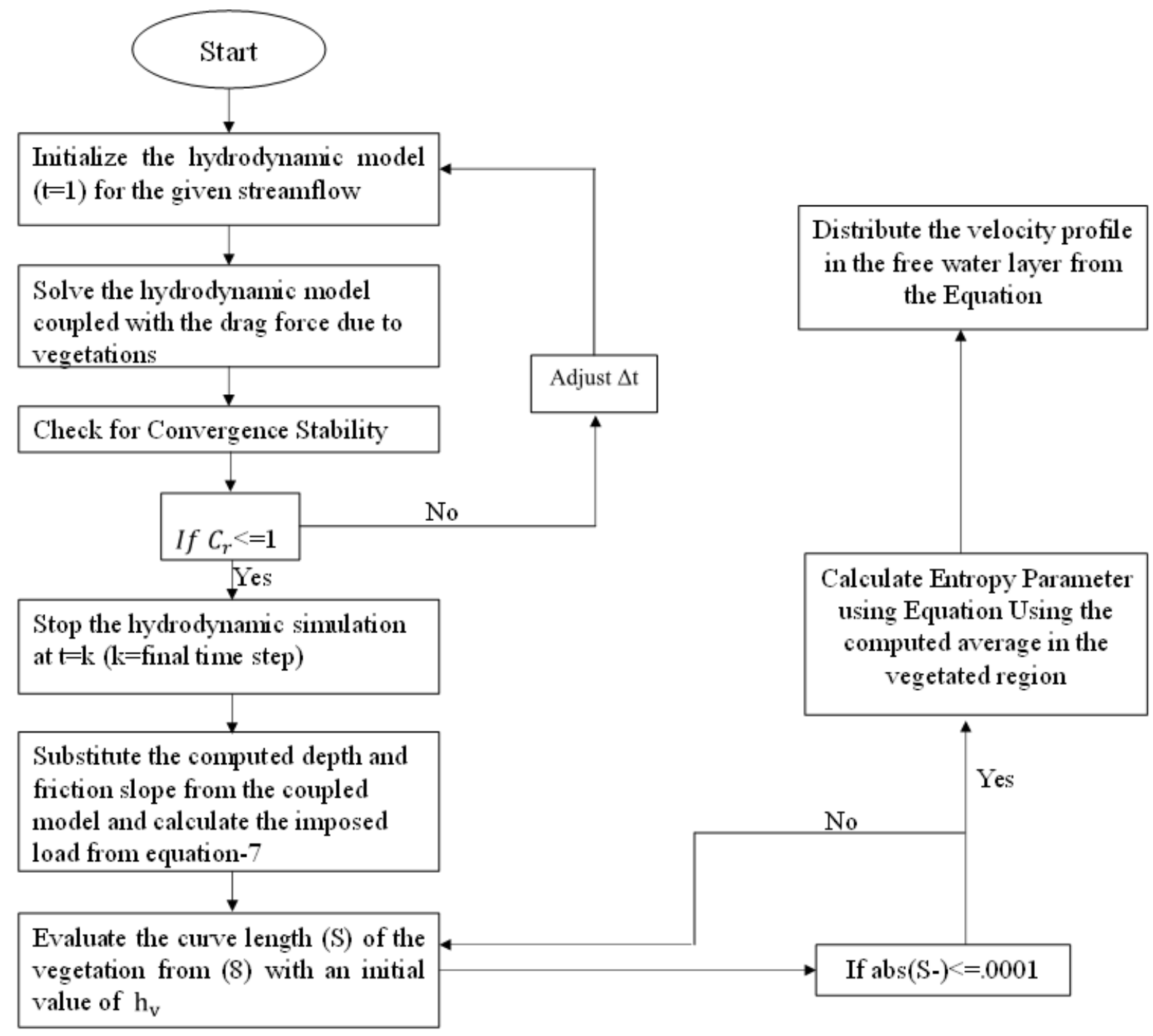

Figure 3 : Flowchart of the model 

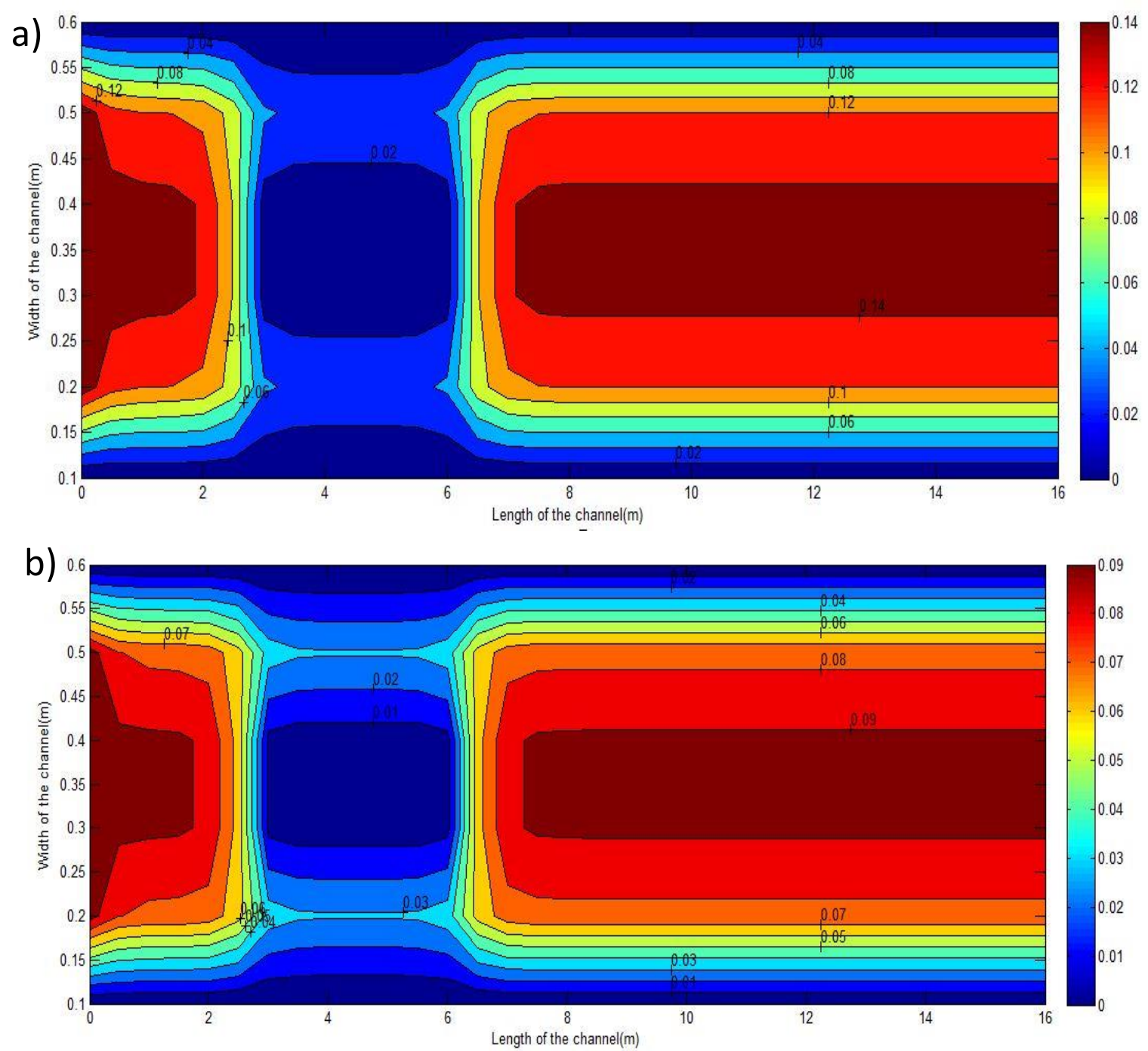

Figure-4 Computed depth averaged velocity profile for experiment (a) 1.2.1 (b) 4.1.1 


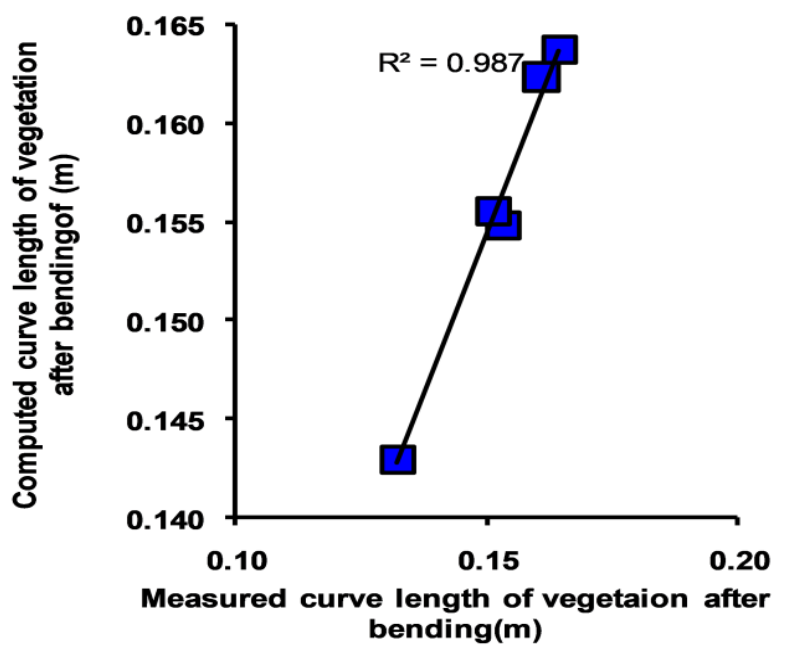

Figure-5: Correlation coefficient between the measured and predicted curve length of the flexible stem 

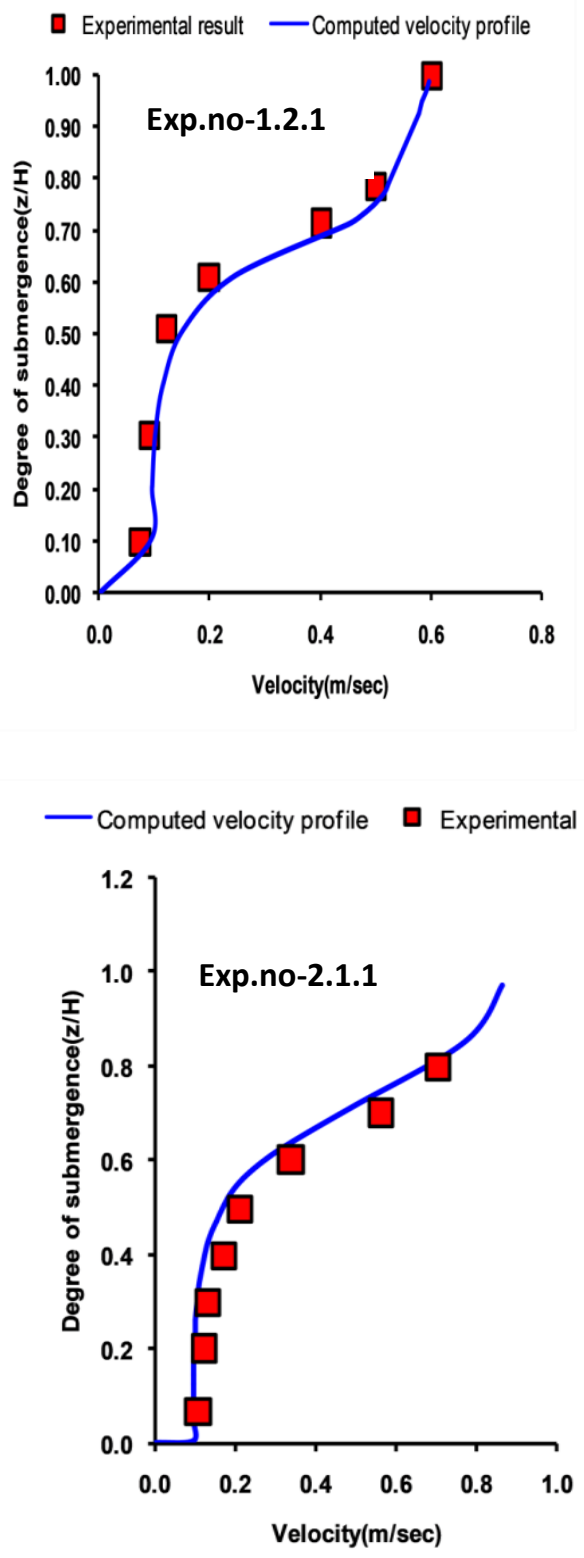

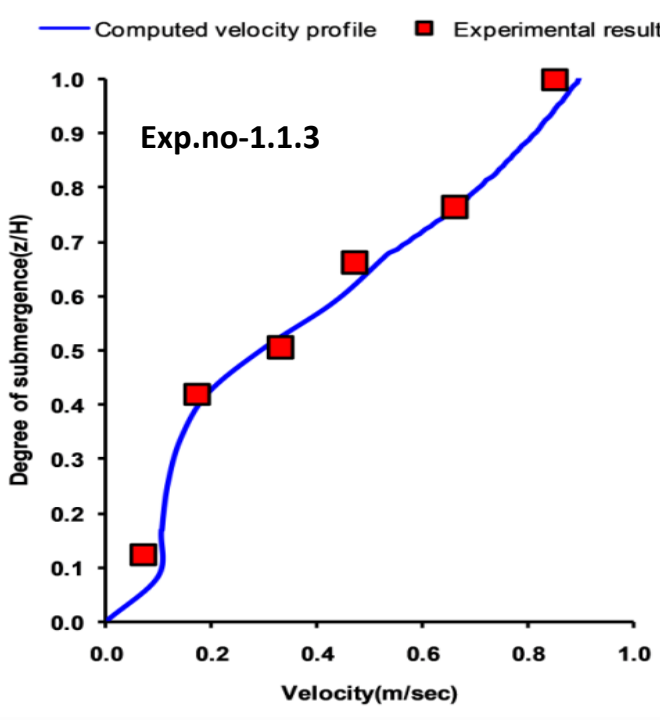

口 Experimental result -Computed velocity profile

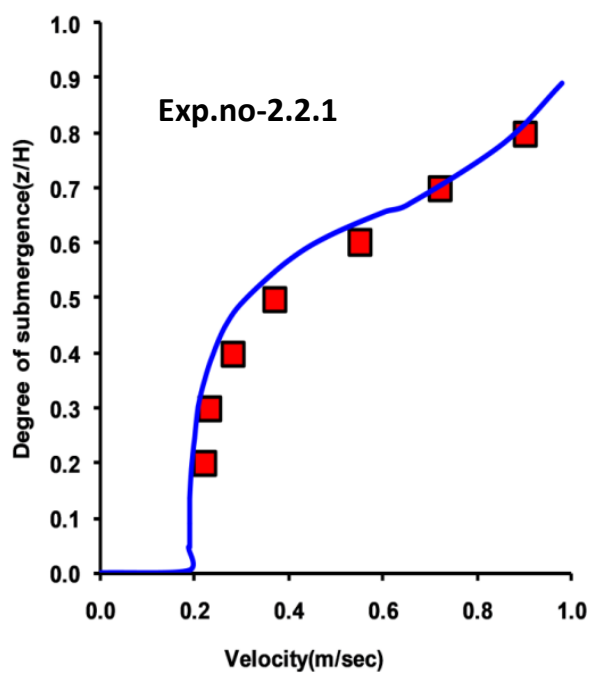


口 Experimental results

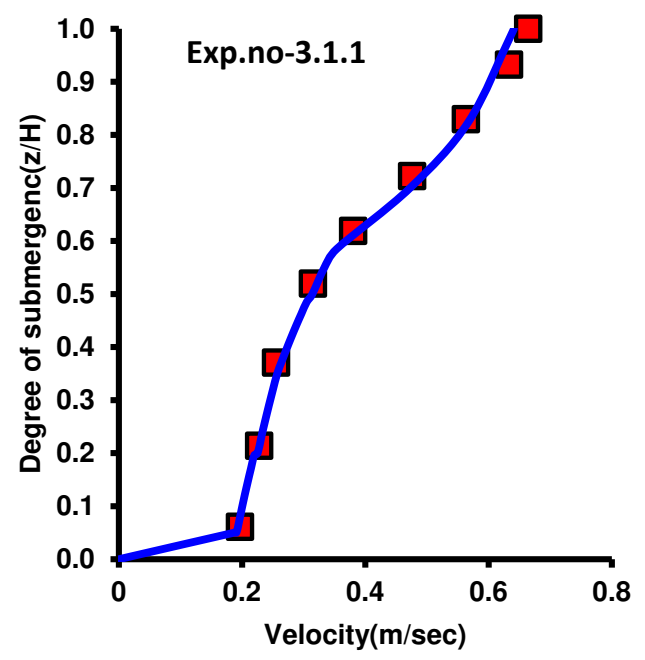

Experimental results - Computed velocity profile

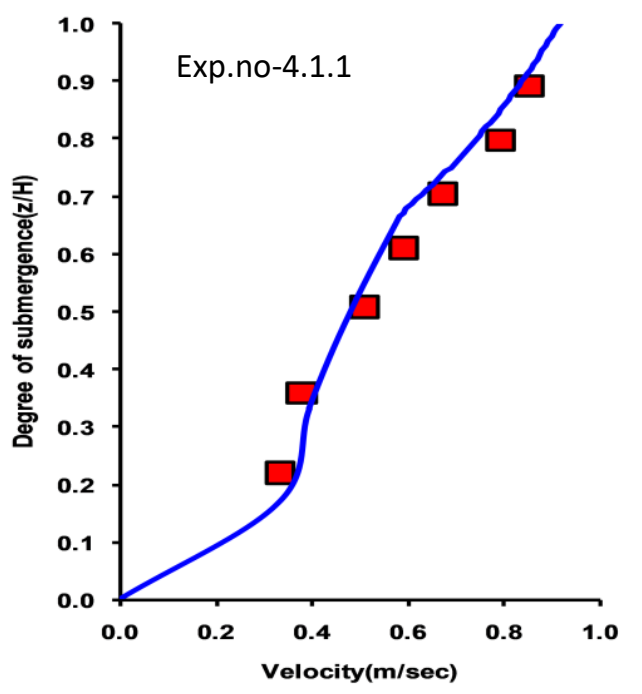

Experimental results $\longrightarrow$ Computed velocity profile

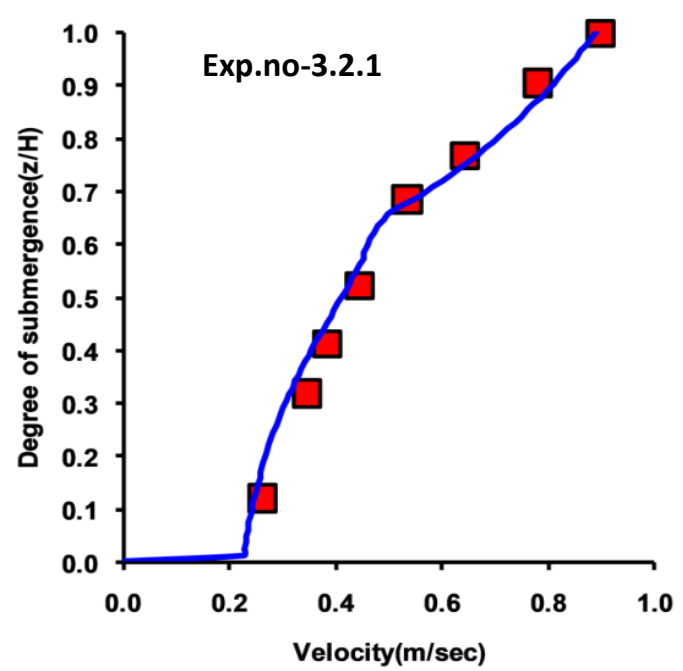

Figure 6: Computed and Measured vertical velocity profile 
